\title{
Er-Al-Co 块体非晶合金的磁热效应
}

惠希东, 许志一, 吴渊, 陈晓华, 刘雄军, 吕昭平

北京科技大学新金属材料国家重点实验室, 北京 100083

E-mail: xdhui@ustb.edu.cn

2011-07-18 收稿, 2011-09-09 接受

国家重点基础研究发展计划(2007CB613901)和国家自然科学基金(50871013, 51071018)资助项目

摘要 利用水冷铜模铸造法制备了一系列不同成分的 Er-Al-Co 三元块体非晶合金, 研究了该 系列合金的非晶形成能力和磁热效应. 结果表明, 在磁场诱发下, 该系列非晶合金在 $9 \mathrm{~K}$ 左右 发生了顺磁-铁磁性二级磁性转变. 由于具有较高的有效磁子数, 该系列非晶合金具有优异的 磁热效应, 其中 $\mathrm{Er}_{56} \mathrm{Al}_{24} \mathrm{Co}_{20}$ 非晶合金最大磁熵变和磁致冷能力分别为 $16.06 \mathrm{~J} \mathrm{~kg}^{-1} \mathrm{~K}^{-1}$ 和 465.7 $\mathrm{J} \mathrm{kg}^{-1}$, 是氢液化区有竞争力的磁致冷候选材料.

\section{关键词}

Er-Al-Co 块体

非晶合金

磁热效应

居里温度

致冷能力 磁热效应(magnetocaloric effect, MCE)在非晶态 材料中的早期工作多数集中于通过快速凝固获得的 非晶薄带, 主要研究含稀土或过渡金属元素的非晶 合金. 结果表明, 它们的磁性转变温区比较宽, 多数 $\mathrm{RE}_{1-x} M_{x}$ 型合金 $(M$ 为过渡元素)都表现为传统 MCE, 即等温磁熵变为正值. 例如, $\mathrm{Gd}_{70} \mathrm{Fe}_{30}$ 非晶 ${ }^{[1]}$ 在 10 kOe $(10 \mathrm{kOe}=795.775 \mathrm{kA} / \mathrm{m})$ 外场下的最大磁熵变 $\left(-\Delta S_{\max }\right)$ 为 $1.5 \mathrm{~J} \mathrm{~kg}^{-1} \mathrm{~K}^{-1}$. 当 $\mathrm{Fe}$ 被 $\mathrm{Ni}$ 部分取代形成 $\mathrm{Gd}_{70} \mathrm{Fe}_{12} \mathrm{Ni}_{18}$ 非晶时, 其 $-\Delta S_{\text {max }}$ 对应的温度从 287.5 降 低为 $170 \mathrm{~K}$, 在 $70 \mathrm{kOe}$ 下的 $-\Delta S_{\max }$ 为 $7.71 \mathrm{~J} \mathrm{~kg}^{-1} \mathrm{~K}^{-1}$; 而当 $\mathrm{Fe}$ 完全被 $\mathrm{Ni}$ 取代形成 $\mathrm{Gd}_{70} \mathrm{Ni}_{30}$ 非晶合金 ${ }^{[1,2]}$ 时, 居里温度为 $126.3 \mathrm{~K}$, 在 10 和 $70 \mathrm{kOe}$ 下的 $-\Delta S_{\max }$ 分 别为 2.45 和 $1.5 \mathrm{~J} \mathrm{~kg}^{-1} \mathrm{~K}^{-1}$.

具有磁热效应的块体非晶合金(bulk metallic glasses, BMGs)的研究较晚但发展迅速. 2002 年, Shen 等人 ${ }^{[3]}$ 报道了 $\mathrm{Pd}_{40} \mathrm{Ni}_{22.5} \mathrm{Fe}_{17.5} \mathrm{P}_{20}$ 块体非晶合金的磁热 效应, 该合金随温度降低逐渐呈超顺磁、铁磁和自旋 玻璃态, 超顺磁-铁磁转变温度为 $94 \mathrm{~K}, 50 \mathrm{kOe}$ 下的 $-\Delta S_{\max }$ 为 $0.58 \mathrm{~J} \mathrm{~kg}^{-1} \mathrm{~K}^{-1} .2006$ 年, Luo 等人 ${ }^{[4]}$ 研究了 $\mathrm{Gd}$ 基块体非晶的 $\mathrm{MCE}$, 所涉及的成分是 $\mathrm{Gd}_{33} \mathrm{Er}_{22} \mathrm{Al}_{25} \mathrm{Co}_{20}$ 和 $\mathrm{Gd}_{53} \mathrm{Al}_{24} \mathrm{Co}_{20} \mathrm{Zr}_{3}$, 其居里温度分别 为 52 和 $93 \mathrm{~K}$, 在 $50 \mathrm{kOe}$ 下的 $-\Delta S_{\max }$ 分别为 9.47 和
$9.4 \mathrm{~J} \mathrm{~kg}^{-1} \mathrm{~K}^{-1} .2007$ 年, Luo 等人 ${ }^{[5]}$ 又研究了 $\mathrm{Ho}, \mathrm{Dy}$, $\mathrm{Er}$ 基块体非晶合金的 MCE, 报道在零场冷却条件下 这些合金伴随有自旋玻璃 - 顺磁转变, 其中 $\mathrm{Er}_{50} \mathrm{Al}_{24} \mathrm{Co}_{20} \mathrm{Y}_{6}$ 的居里温度为 $8 \mathrm{~K}, 50 \mathrm{kOe}$ 下的 $-\Delta S_{\text {max }}$ 为 $15.91 \mathrm{~J} \mathrm{~kg}^{-1} \mathrm{~K}^{-1} .2008$ 年, $\mathrm{Jo}$ 等人 ${ }^{[6]}$ 研究了 $\mathrm{Gd}_{55} \mathrm{Co}_{20} \mathrm{Fe}_{5} \mathrm{Al}_{20}$ 的 $\mathrm{MCE}$, 发现 $-\Delta S_{\text {max }}$ 出现在 $125 \mathrm{~K}$, 比其居里温度 $130 \mathrm{~K}$ 稍低, $20 \mathrm{kOe}$ 下的 $-\Delta S_{\text {max }}$ 为 $2.24 \mathrm{~J} \mathrm{~kg}^{-1} \mathrm{~K}^{-1}$. 近年来, 本课题组先后报道了 $\mathrm{Gd}_{56-x}$ $\mathrm{Dy}_{x} \mathrm{Al}_{24} \mathrm{Co}_{20}(x=16,20,22)^{[7]}, \mathrm{Gd}_{36} \mathrm{Y}_{20} \mathrm{Al}_{24} \mathrm{Co}_{20}{ }^{[8]}, \mathrm{Ho}_{36^{-}}$ $\mathrm{Dy}_{20} \mathrm{Al}_{24} \mathrm{Co}_{20}{ }^{[9]}, \mathrm{Dy}_{36} \mathrm{Ho}_{20} \mathrm{Al}_{24} \mathrm{Co}_{20}{ }^{[10]}, \mathrm{Er}_{36} \mathrm{Ho}_{20} \mathrm{Al}_{24}$ $\mathrm{Co}_{20}{ }^{[11]}$ 的 $\mathrm{MCE}$, 其中 $\mathrm{Gd}_{40} \mathrm{Dy}_{16} \mathrm{Al}_{24} \mathrm{Co}_{20}$ 在 $50 \mathrm{kOe}$ 外 场下的最大等温磁熵变为 $15.78 \mathrm{~J} \mathrm{~kg}^{-1} \mathrm{~K}^{-1}$.

客观地说, 非晶态合金在相同磁场和温度范围 内的 $-\Delta S_{\text {max }}$ 还是不能跟晶态合金相比拟的. 许多具 有巨磁热效应晶态材料的 $-\Delta S_{\text {max }}$ 之所以较大, 是因 为在磁场诱导下发生了伴随结构变化的一级磁转变, 而已报道的非晶态合金的磁转变都是二级转变, “点 阵”对磁场诱导下的热效应没有贡献. 晶态材料在 MCE 与温度之间的曲线有一个尖锐的峰, 相比之下, 非晶态合金的适用温度范围很宽, 更适合于实现卡 诺循环. 现在要突破的问题是, 希望通过成分设计制 备出磁熵变值能够与典型晶态材料相媲美的材料,

英文版见: Hui X D, Xu Z Y, Wu Y, et al. Magnetocaloric effect in Er-Al-Co bulk metallic glasses. Chinese Sci Bull, 2011, 56, doi: 10.1007/s11434011-4855-7 
并发挥非晶态材料的独特优势.

尽管顺磁盐在低温区已经得到了实际应用, 晶 态顺磁盐, 如钝镓石榴石 (Gadolinium Gallium Garnet, $\mathrm{GGG})$ 的使用温度上限只有 $10 \mathrm{~K}^{[12]}, 10 \mathrm{~K}$ 之后其致冷 能力几乎为零. 稀土元素 $\mathrm{Er}$ 的居里温度为 $20 \mathrm{~K}$, 是 重稀土元素中居里温度最低的. 如上所述, $\mathrm{Er}$ 基块体 非晶合金的居里温度都很低, 刚好处在氢液化的温 度区间, 非晶态合金的致冷温区一般都比较宽, 同时 也已经发现在 $\mathrm{Er}$ 基块体非晶合金中确实能获得较大 的 $-\Delta S_{\text {max }}$, 因此, 通过发现新的 $E r$ 基合金成分来调节 和改进非晶态材料的磁热效应, 并突破顺磁盐适用 温区狭窄的瓶颈, 对于低温致冷工程和低温物理学 发展是有价值的.

\section{1 实验方法}

本研究设计了一系列不同成分的 Er-Al-Co 三元 合金, 另外考虑到磁性元素的浓度越高越有利于磁 热效应, 本工作中 $\mathrm{Er}$ 元素的含量都设计为 $56 \%$ 以上, 所设计的成分如图 1 所示.

采用纯度为 $99.95 \%$ 以上的 $\mathrm{Er}, \mathrm{Al}$ 和 $\mathrm{Co}$ 为原料, 先在电弧炉内熔炼成中间合金, 用 $\mathrm{Ti}$ 锭吸氧, 然后 采用水冷铜模法吸铸成直径为 $225 \mathrm{~mm}$ 的棒状样品. 利用 Rigaku D/max-3B ( $\mathrm{Cu}$ 靶)型 X-射线衍射仪分析 样品的结构, 采用 Netzsch-STA-449C 示差扫描量热 仪分析所制备合金的热效应. 利用美国 Quantum Design 公司的综合物性测量系统 PPMS 9 测试样品的

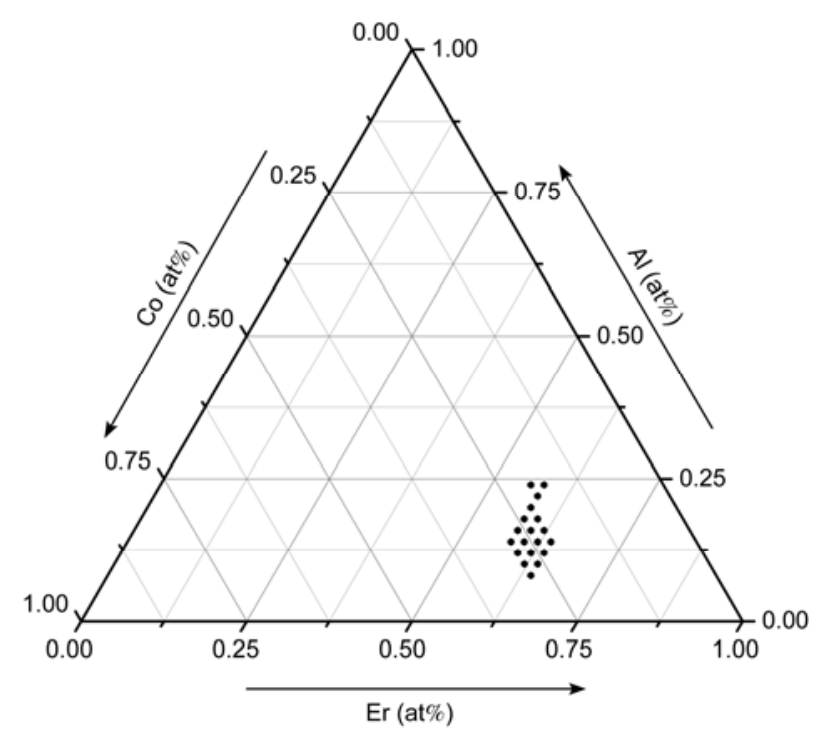

图 1 Er-Al-Co 合金成分设计
磁性能. 测量时, 取非晶样品约 $5 \mathrm{mg}$, 在 200 Oe 磁 场中对样品进行冷场 (即 $\mathrm{FC}$ ) 模式测试, 实验所采用 的冷却速率为 $1 \mathrm{~K} \mathrm{~min}^{-1}$, 测试温度范围 2 50 K, 实 验过程中记录磁化强度与温度之间的变化关系.

\section{2 结果与分析}

直径 2 5 mm 的 Er-Al-Co 合金试棒断面的 XRD 图谱如图 2 所示. 从图中可以看出, 除了成分为 $\mathrm{Er}_{60^{-}}$ $\mathrm{Al}_{12} \mathrm{Co}_{28}$ 和 $\mathrm{Er}_{60} \mathrm{Al}_{16} \mathrm{Co}_{24}$ 合金的 XRD 图谱上有明显的 晶体衍射峰, 是非晶基体与晶体相的复相结构, 其他 合金的衍射特征均呈现出在 $35^{\circ}$ 左右出现大的弥散散 射和 $60^{\circ}$ 左右出现小的弥散散射峰, 它们都可以看作 完全非晶结构, 个别有极微弱的晶体衍射峰表明有 少量 $(\approx 5 \%)$ 晶体分布在非晶基体上. 由图可知, $\mathrm{Er}_{56^{-}}$ $\mathrm{Al}_{24} \mathrm{Co}_{20}$ 合金能够得到直径为 $5 \mathrm{~mm}$ 的非晶样品.

对 XRD 曲线上无明显晶化峰的样品进行 DSC 测 试分析, 结果如图 3 所示. 可见随着加热温度的升高, 合金中出现了明显的对应于玻璃转变的吸热反应和 随后若干个对应于晶化反应的放热峰, 这也进一步 证实了它们基本为非晶态结构. 其中, $\mathrm{Er}_{56} \mathrm{Al}_{24} \mathrm{Co}_{20}$ 非 晶合金的过冷液相区宽度 $\left(\Delta T_{x}\right)$ 最大, 可达 $52.2 \mathrm{~K}$, 如 果仅以 $\Delta T_{x}$ 作为评价非晶形成能力的参考指标, 对比 图 2 和 3 可知, $\mathrm{Er}_{56} \mathrm{Al}_{24} \mathrm{Co}_{20}$ 具有较高的非晶形成能力.

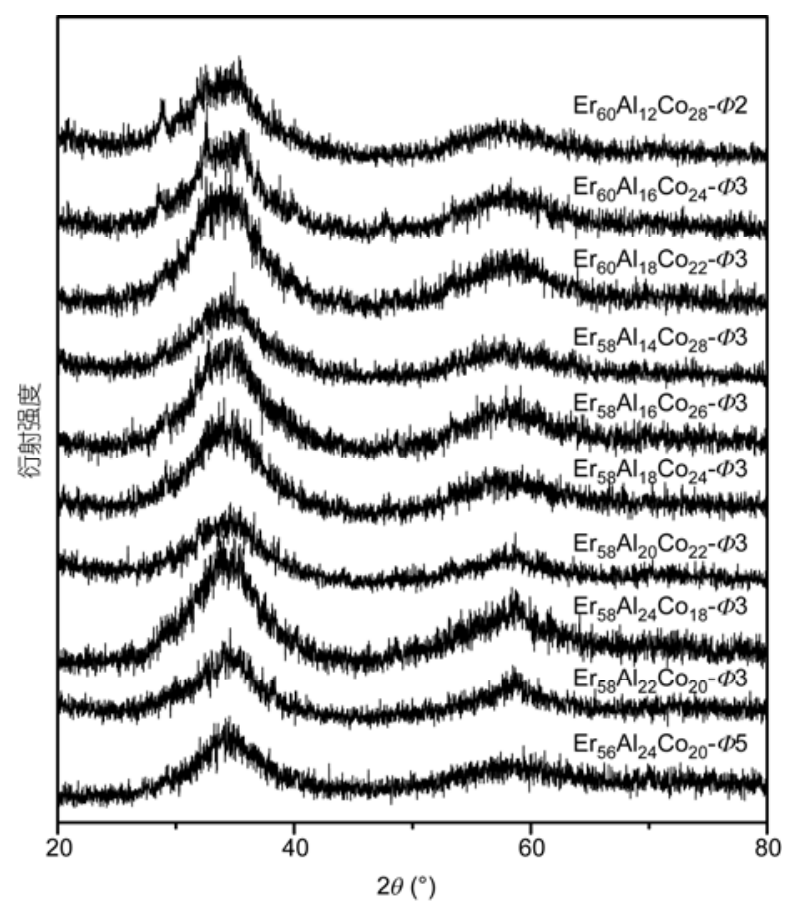

图 2 Er-Al-Co 合金的 XRD 图谱 


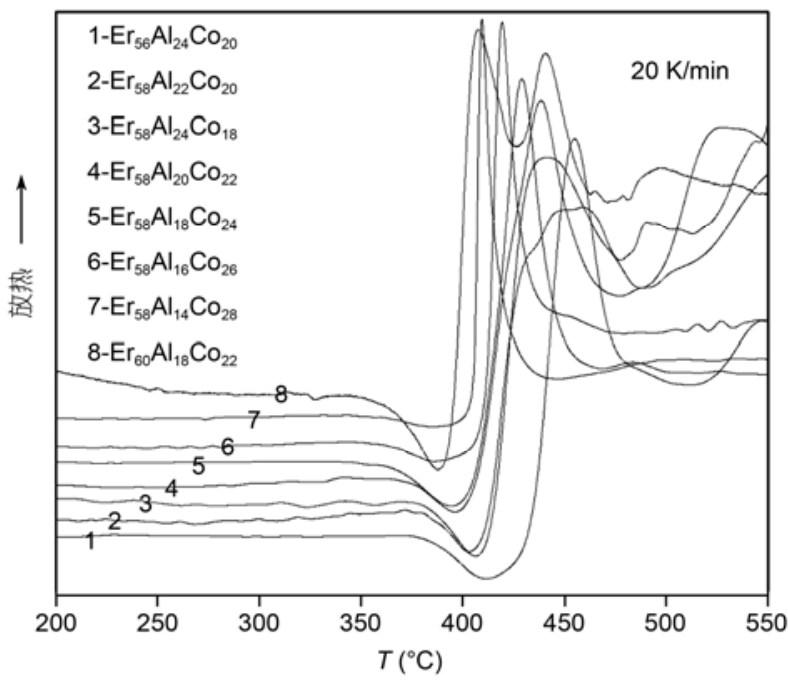

图 3 Er-Al-Co 合金的 DSC 曲线

图 4 给出了 $\mathrm{Er}_{56} \mathrm{Al}_{24} \mathrm{Co}_{20}, \mathrm{Er}_{58} \mathrm{Al}_{22} \mathrm{Co}_{20}, \mathrm{Er}_{58} \mathrm{Al}_{24}-$ $\mathrm{Co}_{18}$ 和 $\mathrm{Er}_{58} \mathrm{Al}_{14} \mathrm{Co}_{28}$ (以下分别表示为 $\mathrm{E} 1, \mathrm{E} 2, \mathrm{E} 3$ 和 $\mathrm{E} 4)$ 的磁温曲线, 图 5 则相应给出了用于确定居里温 度 $\left(T_{\mathrm{c}}\right)$ 的微分曲线, $T_{\mathrm{c}}$ 对应于微分曲线峰谷温度值. 从图 4 和 5 可见, 磁化强度在临界温度附近的变化是 非常急剧的, 根据 Maxwell 关系可以推断, 这些合金
在 $T_{\mathrm{c}}$ 附近可能具有较大的 MCE. 从图 5 中可以标出 $\mathrm{E} 1 \sim \mathrm{E} 4$ 合金的 $T_{\mathrm{c}}$ 值分别为 $8.5,8.6,8.5$ 和 $8.6 \mathrm{~K}$, 也可以 看到这些成分不同的合金具有相近的 $T_{\mathrm{c}}$. 这些非晶合 金的 $T_{\mathrm{c}}$ 比稀土元素 $\mathrm{Er}$ 的低 $10 \mathrm{~K}$ 以上, 与晶态顺磁盐 GGG 的 $T_{\mathrm{c}}$ 非常接近, 因此适合在液氢温度区间工作.

非晶合金的 $T_{\mathrm{c}}$ 相对于纯 $\mathrm{Er}$ 大幅度的减少可以从 分子场理论进行分析, 按照分子场理论, 铁磁体的

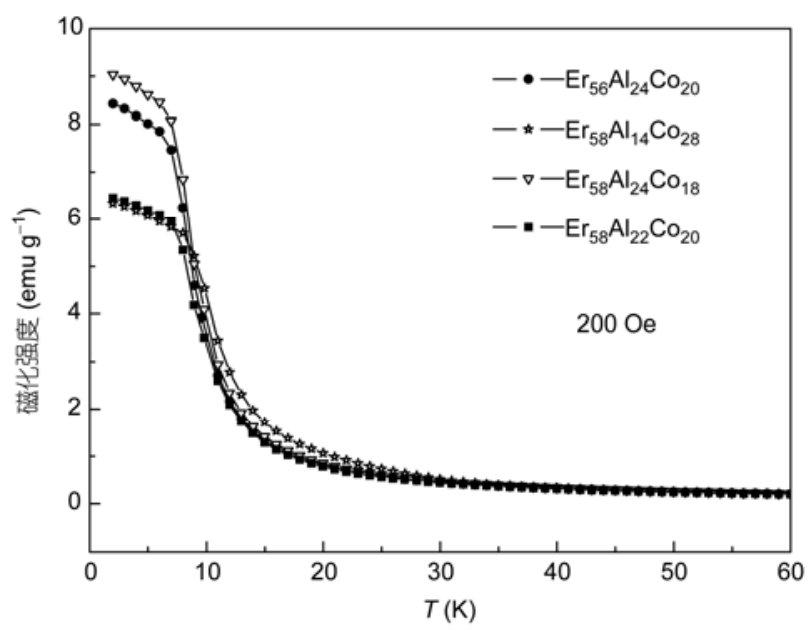

图 4 Er-Al-Co BMGs 在 200 Oe 外场下的磁温曲线

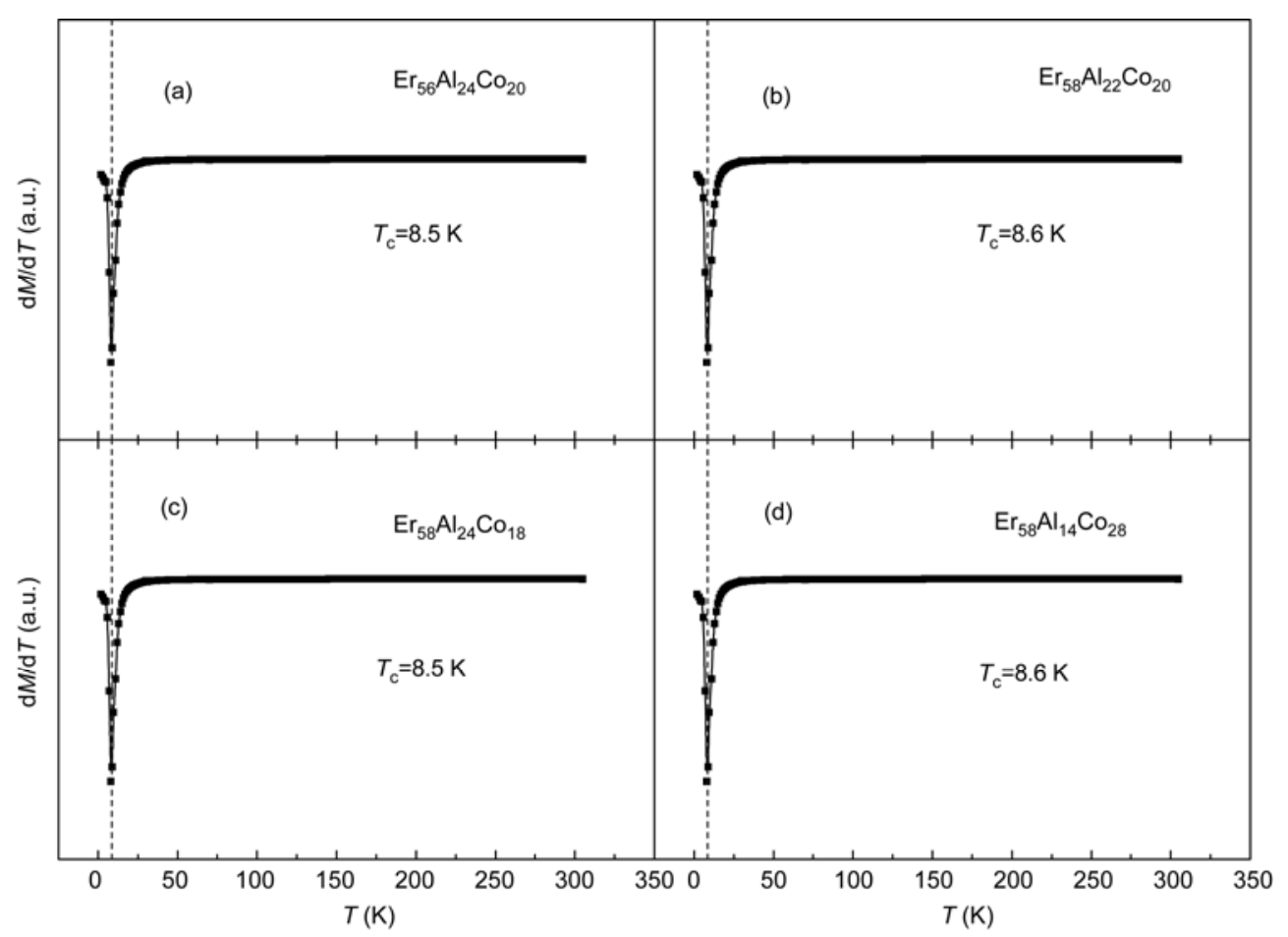

图 5 Er-Al-Co BMGs 在 200 Oe 外场下测得的居里温度 
$T_{\mathrm{c}}^{[13]}$ 可以表达为

$$
T_{\mathrm{c}}=\frac{2 Z A_{\mathrm{ex}} J(J+1)}{3 k_{\mathrm{B}}},
$$

其中, $Z$ 为配位数, $A_{\mathrm{ex}}$ 为交换积分, $J$ 为总的角动量, $k_{\mathrm{B}}$ 为 Boltzmann 常数. $Z$ 是一个大于或等于晶态结构的 数, 对于非晶态结构常常固定为 $12^{[14]}$. 所以 $T_{\mathrm{c}}$ 的下 降只可能是交换积分或角动量上的不同. 一方面, 通 过合金化作用, $T_{\mathrm{c}}$ 会降低; 另一方面, 由于形成了非 晶态结构，尽管存在局域的浓度起伏，也即存在局域 的交换积分起伏 ${ }^{[2]}$, 非晶态结构的形成还是导致了磁 性元素间的交换积分或角动量数值上的大幅度减小, 从而引起 $T_{\mathrm{c}}$ 的减小.

对磁化强度-温度曲线进行变换, 如图 6 所示, 可以得到磁化率倒数与温度之间的关系曲线, 从图 中可以看出, 在 $10 \mathrm{~K}$ 以上磁化率倒数基本随温度直 线变化, 符合居里-外斯定律 ${ }^{[15]}$

$$
\chi=\frac{C}{T-\Theta_{\mathrm{f}}} \text {. }
$$

这里 $C=\frac{N p^{2} \mu_{\mathrm{B}}{ }^{2}}{3 k_{\mathrm{B}}}, p$ 为有效磁子数, $N$ 为粒子数密度. 那么在 $1 / \chi-T$ 关系图中, 直线的斜率 $B=1 / C$. 将 $N$ 与
考察对象的摩尔质量 $M$ 联系起来, 可以得到

$$
p^{2}=\frac{3 k_{\mathrm{B}} M}{B N_{\mathrm{A}} \mu_{\mathrm{B}}^{2}} \text {. }
$$

将 $B$ 的单位定为 $\mathrm{Oe} \mathrm{g} \mathrm{emu}^{-1} \mathrm{~K}^{-1}, M$ 的单位定为 $\mathrm{g} \mathrm{mol}^{-1}$, $N_{\mathrm{A}}=6.02 \times 10^{23} \mathrm{~mol}^{-1}, \mu_{\mathrm{B}}=9.3 \times 10^{-21} \mathrm{emu}, k_{\mathrm{B}}=1.38 \times$ $10^{-23} \mathrm{~J} \mathrm{~K}^{-1}, 1 \mathrm{~J}=10^{7}$ Oe emu, 则

$$
p=\sqrt{7.95 \frac{M}{B}}
$$

其中 $M$ 和 $B$ 均取数值，单位已约去，即“分子”的有效 磁矩为 $p \mu_{\mathrm{B}}$. 依据对直线部分的拟合, 计算出来的 $\mathrm{Er}_{56} \mathrm{Al}_{24} \mathrm{Co}_{20}, \mathrm{Er}_{58} \mathrm{Al}_{22} \mathrm{Co}_{20}, \mathrm{Er}_{58} \mathrm{Al}_{24} \mathrm{Co}_{18}$ 和 $\mathrm{Er}_{58} \mathrm{Al}_{14}$ $\mathrm{Co}_{28}$ 的有效磁子数分别为 7.00, 7.03, 7.43 和 7.04. 结 合有效磁子数与最大等温磁熵变之间的关联性, 这 些非晶合金可望具有较高的最大磁熵变.

对这 4 种成分的合金在 0 50 kOe 范围内进行了 不同温度下的等温磁化测试，结果如图 7 所示. 可见， 这些顺磁-铁磁性转变特征非常明显, 它们在低温下 的磁化强度相近. 表明它们的磁熵变也相近. 从图中 还可以看出, 它们的磁化曲线相类似. 为确定磁性转 变的等级, 图 8 绘出了 $\mathrm{Er}_{56} \mathrm{Al}_{24} \mathrm{Co}_{20}$ 的 Arrot 图, 曲线 没有呈现 $\mathrm{S}$ 形，也没有出现斜率为负值的区域，说明
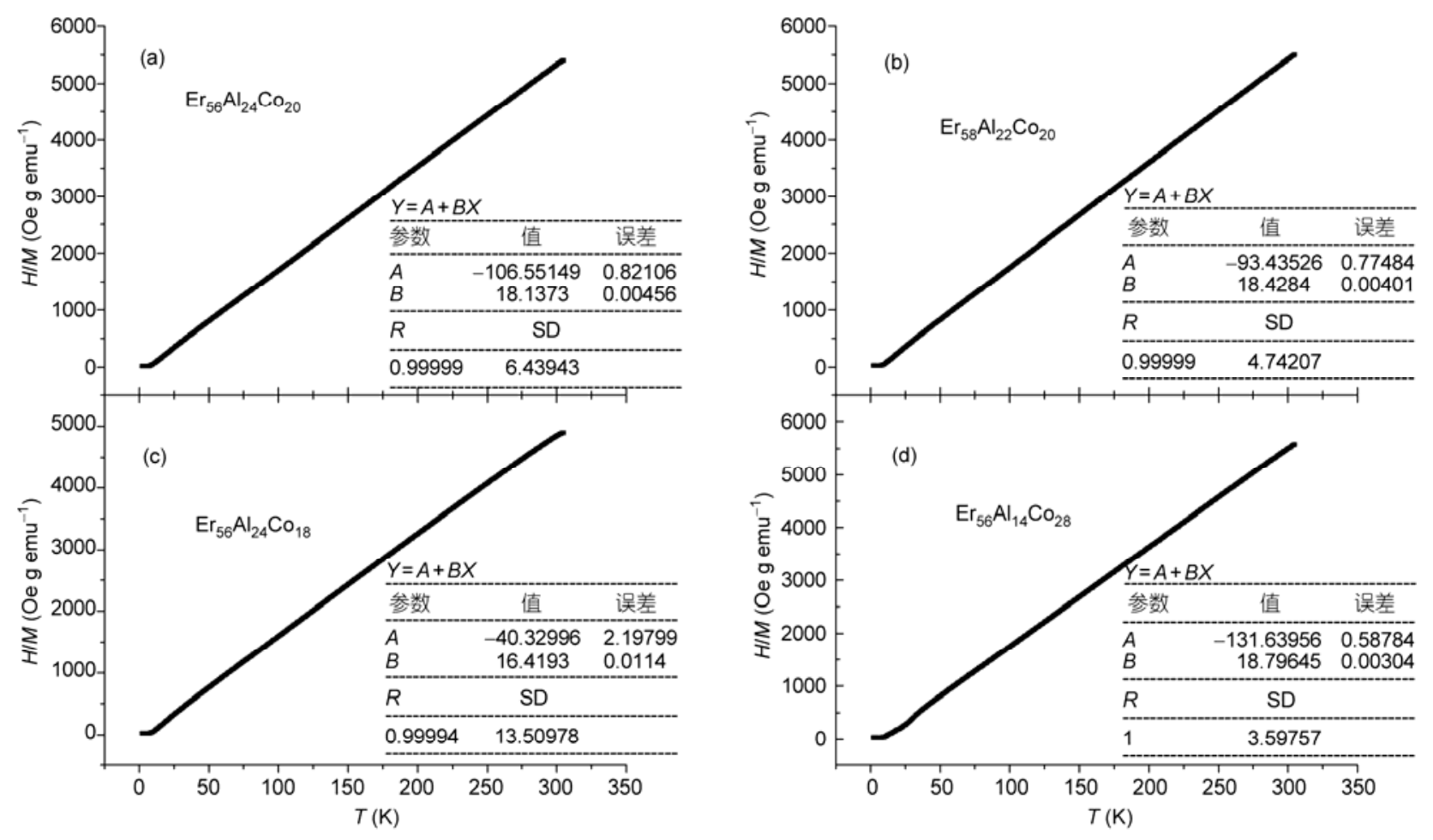

图 6 Er-Al-Co BMGs 在 200 Oe 外场下的 H/M (1/ $\chi)-T$ 图 

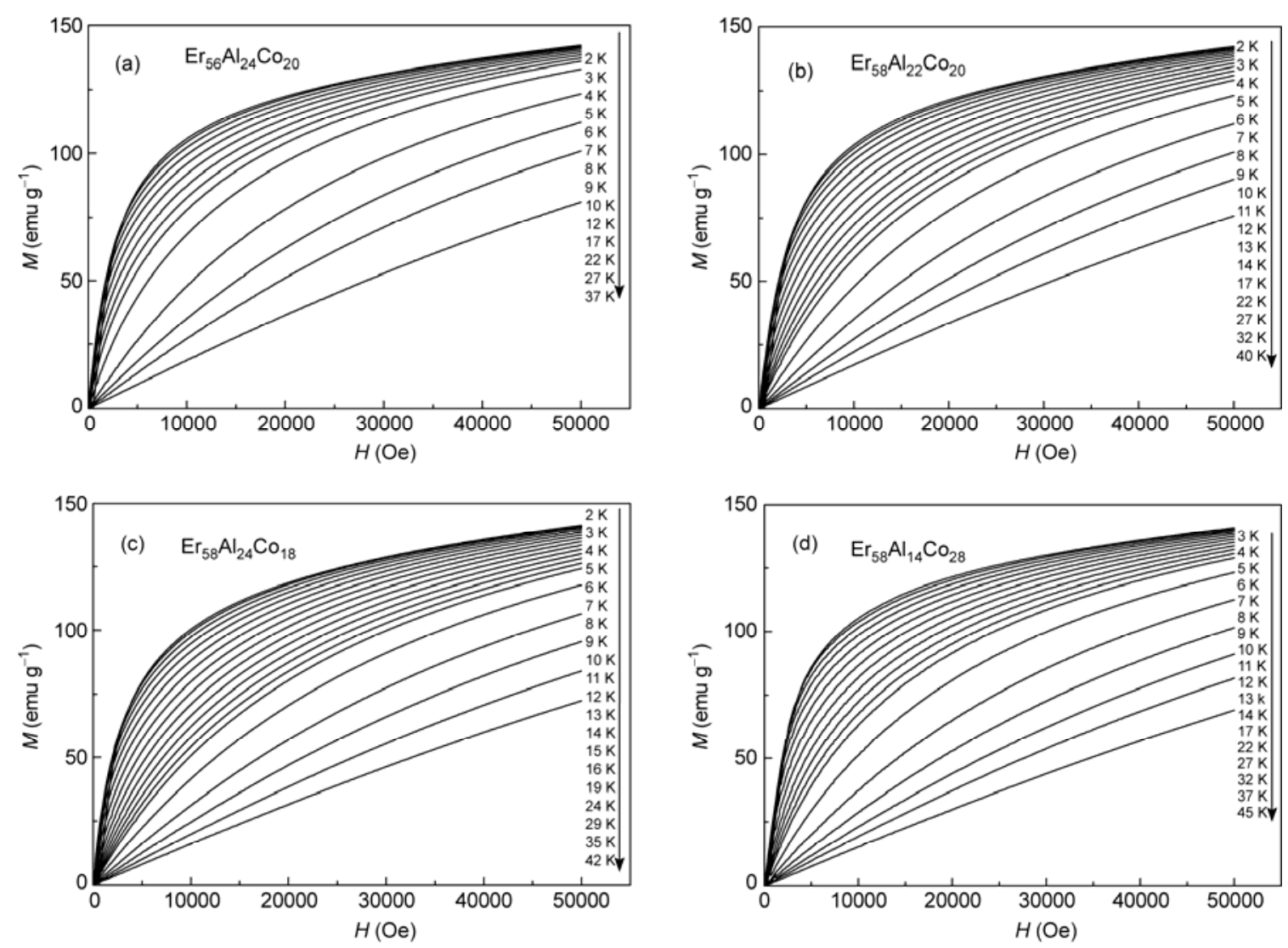

图 7 Er-Al-Co BMGs 在不同温度下的等温磁化曲线

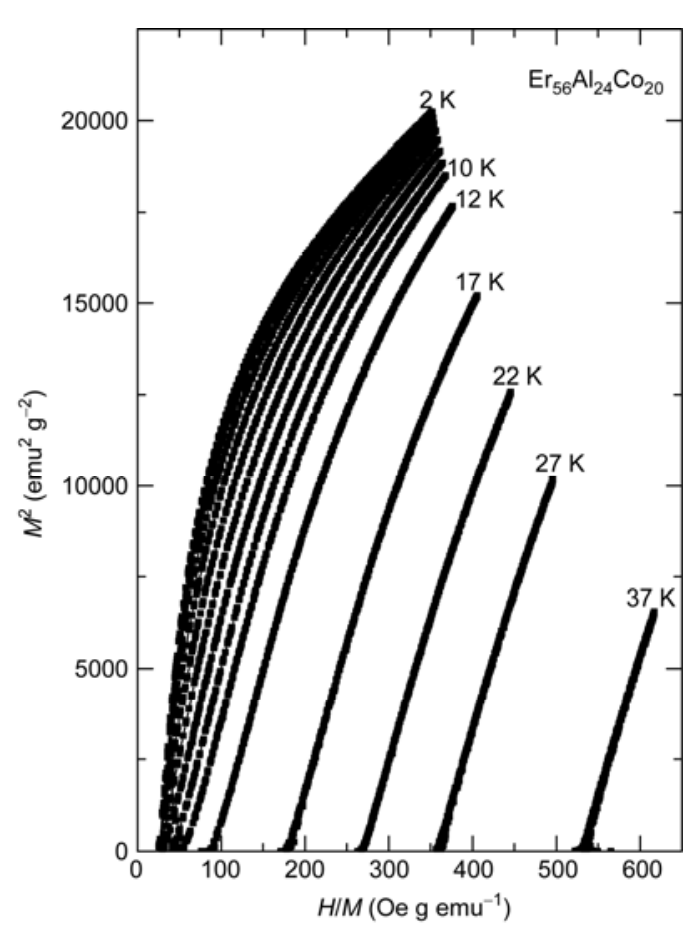

图 $8 \mathrm{Er}_{56} \mathrm{Al}_{24} \mathrm{Co}_{20}$ 的 $\mathrm{Arrot}$ 曲线
磁场诱发下发生的是二级磁性转变.

利用热力学中的 Maxwell关系, 可以推导出磁摘 $S_{\mathrm{M}}$ 、温度 $T$ 、外加磁场的磁感应强度 $B$ 和磁化强度 $M$ 之间的关系为

$$
\left(\frac{\partial S_{\mathrm{M}}}{\partial B}\right)_{T}=\left(\frac{\partial M}{\partial T}\right)_{B}
$$

这里 $B=\mu_{0} H, \mu_{0}$ 为真空磁导率. 在等温过程中, 外加 磁场变化时产生的磁熵变化(等温磁熵变)为

$$
\begin{aligned}
\Delta S_{\mathrm{M}}(T, H) & =S_{\mathrm{M}}(T, H)-S_{\mathrm{M}}(T, 0) \\
& =\int_{0}^{H} \mu_{0}\left[\frac{\partial M(T, H)}{\partial T}\right]_{H} \mathrm{~d} H .
\end{aligned}
$$

在 $50 \mathrm{kOe}$ 外场下计算得到的等温磁熵变随温度 变化的曲线如图 9 所示, 可以看出, E1 E4 合金出现 $-\Delta S_{\max }$ 的温度都高于它们的居里温度, 4 种非晶合金 的 $-\Delta S_{\text {max }}$ 分别为 $16.06,15.71,15.50$ 和 $15.67 \mathrm{~J} \mathrm{~kg}^{-1} \mathrm{~K}^{-1}$. 可见, $\mathrm{E} 1$ 合金的 $-\Delta S_{\max }$ 甚至高于 Liang 等人 ${ }^{[7]}$ 报道的 $\mathrm{Er}_{50} \mathrm{Al}_{24} \mathrm{Co}_{20} \mathrm{Y}_{6}$ 的 $15.91 \mathrm{~J} \mathrm{~kg}^{-1} \mathrm{~K}^{-1}$, 也高于 $\mathrm{Luo}$ 等人 ${ }^{[5]}$ 报道的 $\mathrm{Gd}_{40} \mathrm{Dy}_{16} \mathrm{Al}_{24} \mathrm{Co}_{20}$ 的 $15.78 \mathrm{~J} \mathrm{~kg}^{-1} \mathrm{~K}^{-1}$. 从图 9 


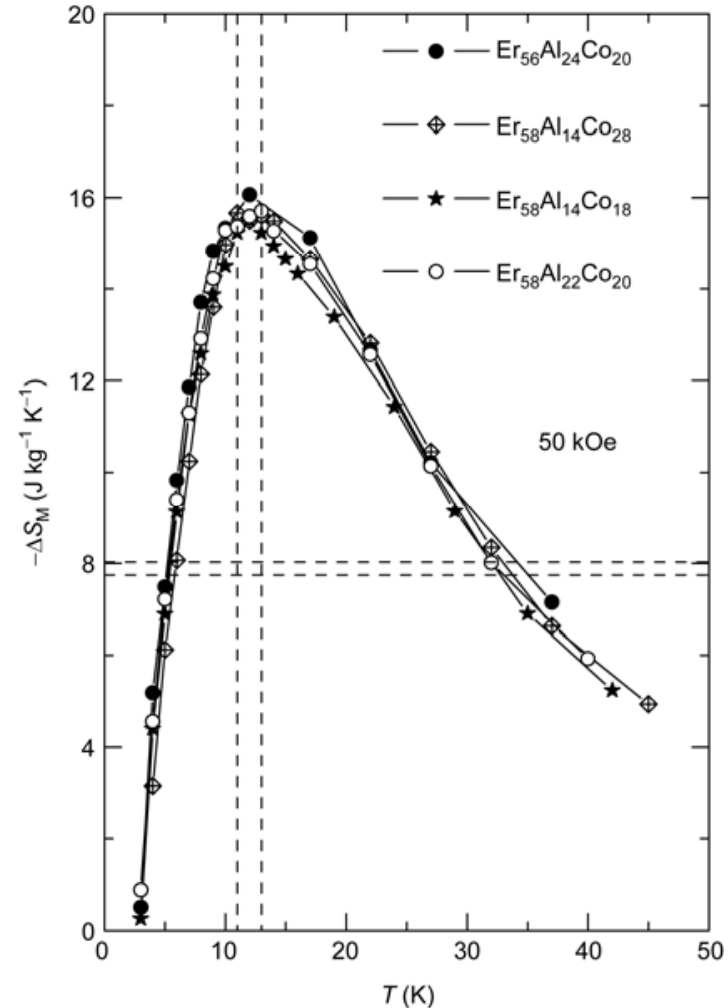

图 9 Er-Al-Co BMGs 在 $50 \mathrm{kOe}$ 下的等温磁熵变

还可知, E1 E4 合金的半高宽温度在 27.5 29 K 之间, 半高宽对应的上限温度区间最高可达 $32 \mathrm{~K}$, 比顺磁 盐的上限温度 $(10 \mathrm{~K}$ 左右) 有大幅提高, 说明它们更适
合于低温下的卡诺循环，是适用于氢液化温度范围 内优异的磁致冷材料. 根据半高宽和最大磁熵变值 的乘积, 可以得到 $\mathrm{E} 1 \sim \mathrm{E} 4$ 合金的磁致冷能力分别为 $465.7,432.0,427.8$ 和 $431.0 \mathrm{~J} \mathrm{~kg}^{-1}$. 这些数据表明, 本 工作制备的 Er-Al-Co 块体非晶合金为磁致冷材料提 供了新的选择.

\section{3 结论}

(1) 利用水冷铜模铸造法制备了一系列不同 成分的 Er-Al-Co 三元块体非晶合金, 通过 XRD 和 DSC 分析, 认为 $\mathrm{Er}_{56} \mathrm{Al}_{24} \mathrm{Co}_{20}$ 合金具有较高非晶形成 能力.

(2) E1 E4 非晶合金的居里温度分别为 8.5, 8.6, 8.5 和 $8.6 \mathrm{~K}$, 有效磁子数分别为 $7.00,7.03,7.43$ 和 7.04. 非晶态结构的形成导致了磁性元素间的交换积 分或角动量数值上的大幅度减小, 从而引起居里温 度的减小. 在磁场诱发下非晶合金发生的是顺磁-铁 磁性二级磁性转变.

(3) $\mathrm{E} 1 \sim \mathrm{E} 4$ 非晶合金的 $-\Delta S_{\text {max }}$ 分别为 $16.06,15.71$, 15.50 和 $15.67 \mathrm{~J} \mathrm{~kg}^{-1} \mathrm{~K}^{-1}$, 磁致冷能力分别为 465.7 , $432.0,427.8$ 和 $431.0 \mathrm{~J} \mathrm{~kg}^{-1}$. $\mathrm{E} 1$ 合金的 $-\Delta S_{\max }$ 高于 Liang 等人报道的 $\mathrm{Er}_{50} \mathrm{Al}_{24} \mathrm{Co}_{20} \mathrm{Y}_{6}$ 的 $-\Delta S_{\max }$, 也高于 Luo 等人报道的 $\mathrm{Gd}_{40} \mathrm{Dy}_{16} \mathrm{Al}_{24} \mathrm{Co}_{20}$ 的 $-\Delta S_{\max }$. 本工作 制备的 Er-Al-Co 块体非晶合金有望成为新的磁致冷 候选材料.

\section{参考文献}

1 Foldeaki M, Gopal B R, Chahine R, et al. Composition dependence of magnetic properties in amorphous rare-earth-metal-based alloys. J Magn Magn Mater, 1997, 174: 295-308

2 Liu X Y, Barclay J A, Gopal B R, et al. Thermomagnetic properties of amorphous rare-earth alloys with Fe, Ni, or Co. J Appl Phys, 1996, 79: $1630-1641$

3 Shen T D, Schwarz R B, Coulter J Y, et al. Magnetocaloric effect in bulk amorphous $\mathrm{Pd}_{40} \mathrm{Ni}_{22.5} \mathrm{Fe}_{17.5} \mathrm{P}_{20}$ alloy. J Appl Phys, 2002, 91: $5240-5245$

4 Luo Q, Zhao D Q, Pan M X, et al. Magnetocaloric effect in Gd-based bulk metallic glasses. Appl Phys Lett, 2006, 89: 081914

5 Luo Q, Zhao D Q, Pan M X, et al. Magnetocaloric effect of Ho-, Dy-, and Er-based bulk metallic glasses in helium and hydrogen liquefaction temperature range. Appl Phys Lett, 2007, 90: 211903

6 Jo C-L, Xia L, Ding D, et al. Glass formation ability, structure and magnetocaloric effect of a heavy rare-earth bulk metallic glassy $\mathrm{Gd}_{55} \mathrm{Co}_{20} \mathrm{Fe}_{5} \mathrm{Al}_{20}$ alloy. J Alloys Compd, 2008, 458: 18-21

7 Liang L, Hui X, Chen G L. Thermal stability and magnetocaloric properties of GdDyAlCo bulk metallic glasses. Mater Sci Eng B, 2008, 147: $13-18$

8 Liang L, Hui X, Wu Y, et al. Large magnetocaloric effect in $\mathrm{Gd}_{36} \mathrm{Y}_{20} \mathrm{Al}_{24} \mathrm{Co}_{20}$ bulk metallic glass. J Alloy Compd, 2008, 457: 541-544

9 Liang L, Hui X, Zhang C M, et al. A novel $\mathrm{Ho}_{36} \mathrm{Dy}_{20} \mathrm{Al}_{24} \mathrm{Co}_{20}$ bulk metallic glass with large magnetocaloric effect. Solid State Commu, 2008, 146: 49-52 
10 Liang L, Hui X, Zhang C M, et al. A Dy- based bulk metallic glass with high thermal stability and excellent magnetocaloric properties. J Alloy Compd, 2008, 463: 30-33

11 Liang L, Hui X, Zhang C M, et al. An Er- based bulk metallic glass with high thermal stability and excellent magnetocaloric properties. Intermetallics, 2008, 16: 198-201

12 McMichael R D, Ritter J J, Shull R D. Enhanced magnetocaloric effect in $\mathrm{Gd}_{3} \mathrm{Ga}_{5-x} \mathrm{Fe}_{x} \mathrm{O}_{12}$. J Appl Phys, 1993, 73: 6946-6948

13 Mathias G. Fundamentals of Magnetism. Berlin: Springer, 2008

14 Mansuripur M, Ruane M F. Mean-field analysis of amorphous rare earth-transition metal alloys for thermomagnetic recording. IEEE Trans Magn, 1986, 22: 33-43

15 Kittel C. 项金钟, 吴兴惠, 译. 固体物理导论. 第 8 版. 北京: 化学工业出版社, 2008

- 动 态

\section{模拟失重环境下个体认知功能研究新进展}

目前, 关于长期在轨飞行中航天员作业能力的研究, 大多数的研究主要集中在失重或模拟失重条件下个体生理 功能的变化规律上. 但是, 面对千变万化的太空世界, 宇 航员要随时监控外部世界和内心活动、实施计划行为、形 成推理、解决问题、同时完成多项任务. 在轨飞行要求宇航 员准确、高效地完成航天器系统运行管理、载荷试验、交会 对接和出舱活动等大量在轨作业任务, 要做到这些, 除了 要求航天员健康的生理体制外, 对航天员的认知能力也提 出极高的要求. 迄今为止, 相对于失重或模拟失重条件下 个体生理功能的研究, 关于个体认知功能的变化规律和影 响机制的研究还不多见.

国际上仅有的一些关于失重或模拟失重条件下个体 认知功能的研究得出了不一致的研究结论: 第一种观点认 为, 真实太空环境下个体的认知功能会受到损害; 第二种 观点则认为, 模拟失重条件下个体的认知功能并未受到损 害. 有关失重或模拟失重条件下个体认知功能是否受损的 研究结论不一致, 原因之一或许在于没有深入考察第三变 量的影响.
针对这一问题, 北京师范大学周仁来教授课题组与我 国航天员训练中心合作, 采用工作记忆 2-back 任务、Beck 焦虑量表和 Beck 抑郁自评量表对 $-6^{\circ}$ 头低位卧床期间个体 的工作记忆能力以及情绪状态进行同步地记录, 进一步深 人、系统地揭示航天环境下个体认知功能的变化规律, 试 图揭示以往对真实太空环境和模拟失重环境条件下, 个体 认知功能是否受损研究结果不一致的原因. 研究结果发 现，卧床期间被试的工作记忆成绩变化趋势与对照组一 致, 在 $-6^{\circ}$ 头低位卧床模拟失重条件下, 被试并未出现工作 记忆能力的损害, 被试在卧床期间并未出现临床焦虑和抑 郁情绪. 研究认为, 与航天特殊环境相比, 在模拟失重条 件下, 个体的认知功能未受损, 这很有可能与头低位卧床 环境未能诱发被试的临床焦虑与抑郁情绪有关. 该研究 得到国家重点基础研究发展计划(2011CB711000)的资助, 相关的研究成果发表于 Acta Astronautica 2011 年第 69 卷上. 